\title{
A Note on High-dimensional D. H. Lehmer Problem
}

\author{
Di Han, Zhefeng Xu*, Yuan Yi and Tianping Zhang
}

\begin{abstract}
By using the properties of trigonometric sums and the estimates of $n$ dimensional Kloosterman sums, we study the high-dimensional D. H. Lehmer problem over incomplete intervals. First we generalize the previous results in [11] by presenting some sharp asymptotic formulae. Then with the aid of a more elementary method, we improve the error terms in a straight-forward manner.
\end{abstract}

\section{Introduction}

Let $q>2$ be an odd integer. If an integer $b$ with $0<b<q$ and $(q, b)=1$ has an inverse $\bar{b}$ modulo $q$ with $2 \nmid(b+\bar{b})$, then $b$ is called a D. H. Lehmer number. Denote the number of all Lehmer numbers modulo $q$ by $M(1, q)$, D. H. Lehmer (see [3, Problem F12, p. 251]) asked whether anything non-trivial could be said about $M(1, p)$, where $p$ is an odd prime. Zhang [16] proved that

$$
M(1, q)=\frac{\phi(q)}{2}+O\left(q^{1 / 2} d^{2}(q) \ln ^{2} q\right),
$$

where $\phi(q), d(q)$ are the Euler and the divisor functions, respectively. Then $\mathrm{Xu}$ and Zhang [12] studied the mean square value of the error term of D. H. Lehmer problem over the incomplete interval $[1,(q-1) / 2]$, which proved that the bound of the error term is best to the possible. While for $q=p$ being an odd prime, Cohen and Trudgian 2 recently made Zhang's work explicit, that is,

$$
\left|M(1, p)-\frac{p-1}{2}\right|<\frac{1}{2} p^{1 / 2} \log ^{2} p .
$$

They [2] also connected the problem with primitive roots.

In [4,5], Khan and Shparlinski studied the maximal difference between an integer and its inverse

$$
M(q)=\max \{|a-\bar{a}|: 1 \leq a \leq q,(a, q)=1\},
$$

Received June 11, 2021; Accepted July 29, 2021.

Communicated by Liang-Chung Hsia.

2020 Mathematics Subject Classification. 11L07, 11L05.

Key words and phrases. D. H. Lehmer problem, Kloosterman sums, asymptotic formula.

This work is supported by the N.S.F. $(11971381,11701447,11871317)$ of China and the Basic Research Program for Nature Science of Shaanxi Province (2014JM1001, 2015KJXX-27, 2017JQ1002, 2021JC-29).

*Corresponding author. 
and proved

$$
q-M(q)=o\left(q^{3 / 4+\epsilon}\right)
$$

for any $\epsilon>0$. Then $\mathrm{Xu}[10$ studied the distribution of the difference of an integer and its $m$-th power modulo $q$ over incomplete intervals $[1,[\lambda q]]$ with $0<\lambda \leq 1$. While Zhang and Liu 13 gave an identity for $2 k$-th power of the non-negative least residue $r_{p}(q)$ and Fermat quotient $q_{p}(q)$ of an integer $q$.

Now let $s$ be a nonnegative integer, and $a, b$ be integers with $0<a, b<q$. Zhang 17 investigated the distribution of $2 s$-th power of $|a-b|$, and obtained that

$$
\sum_{\substack{a=1 \\ a b \equiv 1(\bmod q) \\ 2 \nmid(a+b)}}^{q} \sum_{\substack{b=1 \\ \text { a }}}^{\prime}(a-b)^{2 s}=\frac{\phi(q) q^{2 s}}{(2 s+1)(2 s+2)}+O\left(4^{s} q^{(2 s+1 / 2)} d^{2}(q) \ln ^{2} q\right) .
$$

In addition, $\mathrm{Lu}$ and $\mathrm{Yi}$ [6, 7] generalized the problems in [16, 17]. Let $n \geq 2$ be a fixed integer, $c$ and $q \geq 3$ be integers with $(n, q)=(c, q)=1$. For $0<\lambda_{1}, \lambda_{2} \leq 1$, they obtained

$$
\sum_{\substack{a=1 \\ a b \equiv c(\bmod q) \\ n \nmid(a+b)}}^{\left[\lambda_{1} q\right]} \sum_{\substack{\left[\lambda_{2} q\right] \\ n}}^{\prime} 1=\left(1-\frac{1}{n}\right) \lambda_{1} \lambda_{2} \phi(q)+O\left(q^{1 / 2} d^{6}(q) \ln ^{2} q\right) .
$$

Let $N$ be a positive integer and $\alpha>0$. For any integer $M$, they also derived

$$
\sum_{\substack{a=M+1 \\ a b \equiv c(\bmod q) \\ n \mid(a+b)}}^{M+N} \sum_{b=M+1}^{M+N}|a-b|^{\alpha}=\frac{2 \phi(q)}{(\alpha+1)(\alpha+2) n q^{2}} N^{\alpha+2}+O\left(q^{1 / 2+\varepsilon} N^{\alpha}\left(N q^{-1}+1\right)\right) .
$$

In fact, they studied the more general case.

Another important direction is concerning the high-dimensional case. Let $q>2$ and $c$ are two integers with $(q, c)=1, k$ be a fixed positive integer, then for any nonnegative integers $s$, Zhang and Zhang 15 considered the distribution of $2 s$-th power of $\mid b_{1} \cdots b_{k}-$ $b_{k+1} \mid$, and obtained the asymptotic formula

$$
\sum_{\substack{b_{1}=1 \\ b_{1} \cdots b_{k} b_{k+1} \equiv c(\bmod q)}}^{q} \cdots \sum_{\substack{b_{k}=1 \\ b_{k+1}=1}}^{q} \sum_{\substack{k \\ b_{2}}}^{\prime}\left(b_{1} \cdots b_{k}-b_{k+1}\right)^{2 s}=\frac{\phi^{k}(q) q^{2 s k}}{(2 s+1)^{k}}+O\left(4^{s} q^{(2 s+1) k-1 / 2} d^{2}(q) \ln q\right) .
$$

Soon afterwards, Shparlinski 8 improved the error term to $O\left(2 s q^{(2 s+1) s-s+1}\right)$. Almost at the same time, Alkan, Stan and Zaharescu [1] considered $k+1$-tuples numbers with product congruent to 1 modulo $q$, and the parity condition is replaced by linear congruences with respect to more general moduli. 
Now let $m$ be a nonnegative integer, and $k$ be a fixed positive integer. For any positive integer $t$ with $t \leq k$, it is natural to ask what about the distribution of $m$-th power of $\left(b_{1} \cdots b_{t}-b_{t+1} \cdots b_{k+1}\right)$ over incomplete intervals. To be specific, let $0<\lambda_{1}, \lambda_{2}, \ldots, \lambda_{k+1} \leq$ 1 be real numbers and $\mathbf{w}=\left(\lambda_{1}, \lambda_{2}, \ldots, \lambda_{k+1}\right)$. Let $q \geq \max \left\{\left[1 / \lambda_{j}\right]: j=1,2, \ldots, k+1\right\}$ be a positive integer, and $a, n$ are integers coprime to $q$. Define

$$
N(a, k+1, t, \mathbf{w}, q, m, n)=\sum_{\substack{b_{1}=1 \\ b_{1} b_{2} \cdots b_{k+1} \equiv a(\bmod q) \\ n \nmid\left(b_{1}+b_{2}+\cdots+b_{k+1}\right)}}^{\left[\lambda_{1} q\right]} \sum_{\substack{\left.b_{k+1}=1 \\ \lambda_{2} q\right]}}^{\prime\left[\lambda_{k+1} q\right]}\left(b_{1} \cdots b_{t}-b_{t+1} \cdots b_{k+1}\right)^{m} .
$$

It is obvious that by taking $t=k, m=2 s$ and $n=2$, the above reduces to the case considered by $\mathrm{Xu}$ and Zhang [11]. By applying the properties of trigonometric sums and the estimates of high-dimensional Kloosterman sums, they 11 obtained the asymptotic formula

$$
N(a, k+1, k, \mathbf{w}, q, 2 s, 2)=C(k, \mathbf{w}, s) \phi^{k}(q) q^{2 k s}+O\left(4^{s \varepsilon_{k}} q^{(2 s+1) k-1 / 2} d^{2}(q) \ln ^{2} q\right),
$$

where

$$
C(k, \mathbf{w}, s)= \begin{cases}\frac{\left(\lambda_{1} \cdots \lambda_{k}\right)^{2 s+1} \lambda_{k+1}}{2(2 s+1)^{k}} & \text { if } k \geq 2, \\ \frac{\lambda_{1}^{2 s+2}+\lambda_{2}^{2 s+2}-\left(\lambda_{1}-\lambda_{2}\right)^{2 s+2}}{4(s+1)(2 s+1)} & \text { if } k=1\end{cases}
$$

and $\varepsilon_{k}=\frac{1}{2}\left(1-(-1)^{[1 / k]}\right)$.

Actually, the error terms for $N(a, k+1, k, \mathbf{w}, q, 0,2)$ can be improved to the best possible in some special cases like $\mathbf{w}=(1 / 2,1 / 2, \ldots, 1 / 2)$ and $\mathbf{w}=(1 / 4,1 / 4, \ldots, 1 / 4)$, which are shown in [11, 14] by studying the mean square value of the error terms. It should be pointed out that the latter case holds with a strict condition. While for other cases, the methods in [11, 14] failed to improve the error terms.

This article is outlined in the following way: we first generalize the previous results in 11 by presenting some sharp asymptotic formulae for $N(a, k+1, t, \mathbf{w}, q, m, n)$. Then with the aid of a more elementary method, we improve the error terms in a straight-forward manner.

We will prove the following

Theorem 1.1. For any nonnegative integer $m$, we have the asymptotic formulae

$$
N(a, k+1, t, \mathbf{w}, q, m, n)=\left\{\begin{array}{cl}
\left(1-\frac{1}{n}\right) A(k+1, t, \mathbf{w}, m) \phi^{k}(q) q^{m t} & \\
+O\left(q^{m t+k-1 / 2} d^{2}(q) \ln ^{2} q\right) & \text { if }(k+1) / 2<t \leq k, \\
\left(1-\frac{1}{n}\right) B(k+1, t, \mathbf{w}, m) \phi^{k}(q) q^{m(k-t+1)} & \\
+O\left(q^{m(k-t+1)+k-1 / 2} d^{2}(q) \ln ^{2} q\right) & \text { if } 1 \leq t<(k+1) / 2, \\
\left(1-\frac{1}{n}\right) D(k+1, t, \mathbf{w}, m) \phi^{k}(q) q^{m t} & \\
+O\left(2^{m} q^{m t+k-1 / 2} d^{2}(q) \ln ^{2} q\right) & \text { if } t=(k+1) / 2,
\end{array}\right.
$$


where

$$
\begin{aligned}
A(k+1, t, \mathbf{w}, m) & =\frac{\left(\lambda_{1} \cdots \lambda_{t}\right)^{m+1}\left(\lambda_{t+1} \cdots \lambda_{k+1}\right)}{(m+1)^{t}}, \\
B(k+1, t, \mathbf{w}, m) & =\frac{(-1)^{m}\left(\lambda_{1} \cdots \lambda_{t}\right)\left(\lambda_{t+1} \cdots \lambda_{k+1}\right)^{m+1}}{(m+1)^{k-t+1}}, \\
D(k+1, t, \mathbf{w}, m) & =\sum_{j=0}^{m} C_{m}^{j}(-1)^{j} \frac{\left(\lambda_{1} \cdots \lambda_{t}\right)^{m-j+1}\left(\lambda_{t+1} \cdots \lambda_{k+1}\right)^{j+1}}{((m-j+1)(j+1))^{(k+1) / 2}}
\end{aligned}
$$

are computable constants.

Taking $\mathbf{w}=\mathbf{1}=(1,1, \ldots, 1)$ in Theorem 1.1 , we can get the following result about the high-dimensional D. H. Lehmer problem over complete intervals.

Corollary 1.2. For any nonnegative integer $m$, we have

$$
\begin{aligned}
& N(a, k+1, t, \mathbf{1}, q, m, n) \\
& =\sum_{b_{1}=1}^{q} \sum_{b_{2}=1}^{q} \cdots \sum_{b_{k+1}=1}^{q}\left(b_{1} \cdots b_{t}-b_{t+1} \cdots b_{k+1}\right)^{m} \\
& b_{1} b_{2} \cdots b_{k+1} \equiv a(\bmod q) \\
& n \nmid\left(b_{1}+b_{2}+\cdots+b_{k+1}\right)
\end{aligned}
$$

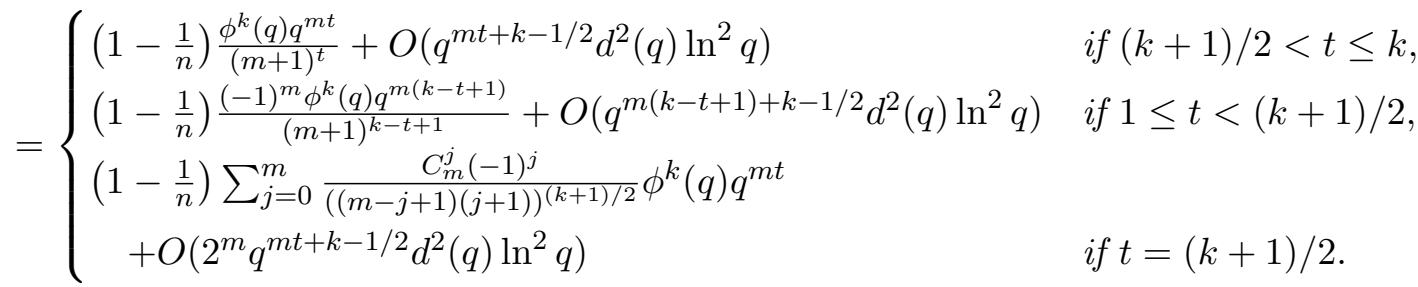

While taking $n=2$ in Theorem 1.1 , we have

Corollary 1.3. For any nonnegative integer $m$, we have the asymptotic formulae

$$
\begin{aligned}
& N(a, k+1, t, \mathbf{w}, q, m, 2) \\
= & \sum_{b_{1}=1}^{\left[\lambda_{1} q\right]\left[\lambda_{2} q\right]} \sum_{b_{2}=1}^{\prime} \cdots \sum_{b_{k+1}=1}^{\left[\lambda_{k+1} q\right]}\left(b_{1} \cdots b_{t}-b_{t+1} \cdots b_{k+1}\right)^{m} \\
& b_{1} b_{2} \cdots b_{k+1} \equiv a(\bmod q) \\
2 \nmid\left(b_{1}+b_{2}+\cdots+b_{k+1}\right) & \\
= & \begin{cases}\frac{A(k+1, t, \mathbf{w}, m)}{2} \phi^{k}(q) q^{m t}+O\left(q^{m t+k-1 / 2} d^{2}(q) \ln ^{2} q\right) & \text { if }(k+1) / 2<t \leq k, \\
\frac{B(k+1, t, \mathbf{w}, m)}{2} \phi^{k}(q) q^{m(k-t+1)}+O\left(q^{m(k-t+1)+k-1 / 2} d^{2}(q) \ln ^{2} q\right) & \text { if } 1 \leq t<(k+1) / 2, \\
\frac{D(k+1, t, \mathbf{w}, m)}{2} \phi^{k}(q) q^{m t}+O\left(2^{m} q^{m t+k-1 / 2} d^{2}(q) \ln ^{2} q\right) & \text { if } t=(k+1) / 2 .\end{cases}
\end{aligned}
$$

Letting $t=k \geq 2$ in Corollary 1.3 , our result recovers the main result of [11]. What's more, taking $k=t=1$ in Theorem 1.1 , we have 
Corollary 1.4. For any nonnegative even integer $m$, we have

$$
\begin{aligned}
N(a, 2,1, \mathbf{w}, q, m, n)= & \sum_{\substack{b_{1}=1 \\
b_{1} b_{2} \equiv a(\bmod q) \\
n \nmid\left(b_{1}+b_{2}\right)}}^{\left[\lambda_{1} q\right]} \sum_{b_{2}=1}^{\left[\lambda_{2} q\right]}\left(b_{1}-b_{2}\right)^{m} \\
= & \left(1-\frac{1}{n}\right) \frac{\lambda_{1}^{m+2}+\lambda_{2}^{m+2}-\left(\lambda_{1}-\lambda_{2}\right)^{m+2}}{(m+1)(m+2)} \phi(q) q^{m} \\
& +O\left(2^{m} q^{m+1 / 2} d^{2}(q) \ln ^{2} q\right) .
\end{aligned}
$$

Taking $n=2, \lambda_{1}=\lambda_{2}=1$ in Corollary 1.4 , we can immediately obtain the result of [17]. While taking $m=0$, we can also obtain the result of [6].

With the notation $\sigma_{m}(\lambda, q)=\sum_{a=1}^{\prime[\lambda q]} a^{m}$, we will give stronger error terms for $N(a, k+$ $1, t, \mathbf{w}, q, m, n)$ in the cases $\mathbf{w}=\left(\lambda_{1}, \lambda_{2}, \ldots, \lambda_{k+1}\right)$ with at least one $\lambda_{j}=1(j=1,2, \ldots, k+$ 1). Define

$$
N_{j}(a, k+1, t, \mathbf{w}, q, m, n)=\sum_{\substack{b_{1}=1 \\ b_{2}=1 \\ b_{1} b_{2} \cdots b_{k+1} \equiv a(\bmod q) \\ n \nmid\left(b_{1}+b_{2}+\cdots+b_{k+1}\right)}}^{\left[\lambda_{1} q\right]} \sum_{\substack{\left.b_{j} \\ b_{2} q\right]}}^{q} \cdots \sum_{\substack{\left.\lambda_{k+1}=1 \\ \lambda_{k+1} q\right]}}^{\prime}\left(b_{1} \cdots b_{t}-b_{t+1} \cdots b_{k+1}\right)^{m} .
$$

Then we have

Theorem 1.5. For any nonnegative integer $m$, we have

$$
\begin{aligned}
N_{j}(a, k+1, t, \mathbf{w}, q, m, n)= & \left(1-\frac{1}{n}\right) \prod_{u=1}^{t} \sigma_{m}\left(\lambda_{u}, q\right) \prod_{\substack{v=t+1 \\
v \neq j}}^{k+1} \sigma_{0}\left(\lambda_{v}, q\right)+O\left(q^{2 k+(m-2) t+1}\right) \\
& +O\left(q^{m t+k / 2} d_{k+1}(q) \ln ^{k+1} q\right) \quad \text { if }(k+1) / 2<t<j, \\
& N_{j}(a, k+1, t, \mathbf{w}, q, m, n) \\
= & \left(1-\frac{1}{n}\right)(-1)^{m} \prod_{\substack{u=1 \\
u \neq j}}^{t} \sigma_{0}\left(\lambda_{u}, q\right) \prod_{v=t+1}^{k+1} \sigma_{m}\left(\lambda_{v}, q\right)+O\left(q^{2 k+(m-2)(k-t+1)+1}\right) \\
& +O\left(q^{m(k-t+1)+k / 2} d_{k+1}(q) \ln ^{k+1} q\right) \quad \text { if } j<t<(k+1) / 2,
\end{aligned}
$$

and

$$
\begin{aligned}
& N_{j}(a, k+1, t, \mathbf{w}, q, m, n) \\
= & \left(1-\frac{1}{n}\right)\left(\prod_{u=1}^{(k+1) / 2} \sigma_{m}\left(\lambda_{u}, q\right) \prod_{\substack{v=(k+3) / 2 \\
v \neq j}}^{k+1} \sigma_{0}\left(\lambda_{v}, q\right)+E(k+1, t, \mathbf{w}, m) \phi^{k}(q) q^{m(k+1) / 2}\right) \\
& +O\left(2^{m} q^{m(k+1) / 2+k-1 / 2} d^{2}(q) \ln ^{2} q\right) \quad \text { if } t=(k+1) / 2<j,
\end{aligned}
$$


where

$$
E(k+1, t, \mathbf{w}, m)=\sum_{j=0}^{m} C_{m}^{j}(-1)^{j} \frac{\left(\lambda_{1} \cdots \lambda_{t}\right)^{m-j+1}\left(\lambda_{t+1} \cdots \lambda_{k}\right)^{j+1}}{((m-j+1)(j+1))^{(k+1) / 2}}
$$

is a computable constant.

Note. Let $q$ be fixed positive integer, then for any nonnegative integer $m$, from the property of Möbius function, we have

$$
\sigma_{m}(\lambda, q)=\sum_{a=1}^{[\lambda q]} a^{m}=\sum_{d \mid q} \mu(d) \sum_{a=1}^{[\lambda q] / d}(a d)^{m}=\frac{[\lambda q]^{m+1}}{m+1} \frac{\phi(q)}{q}+O\left([\lambda q]^{m} 2^{\omega(q)}\right)
$$

This shows the bounds of error terms in the first two cases of Theorem 1.5 are sharper than those of [11. However, our method does not improve the bound of the error term in the case $t=(k+1) / 2$. So how to improve it is still an open problem.

Taking $\mathbf{w}=\mathbf{1}$ in Theorem 1.5 , we may have

Corollary 1.6. For any nonnegative integer $m$, we have

$$
\begin{aligned}
& N(a, k+1, t, \mathbf{1}, q, m, n) \\
& =\sum_{\substack{b_{1}=1 \\
b_{1} b_{2} \cdots b_{k+1} \equiv a(\bmod q) \\
n \nmid\left(b_{1}+b_{2}+\cdots+b_{k+1}\right)}}^{q} \sum_{\substack{b_{k+1}=1 \\
n \nmid}}^{q}\left(b_{1} \cdots b_{t}-b_{t+1} \cdots b_{k+1}\right)^{m}
\end{aligned}
$$

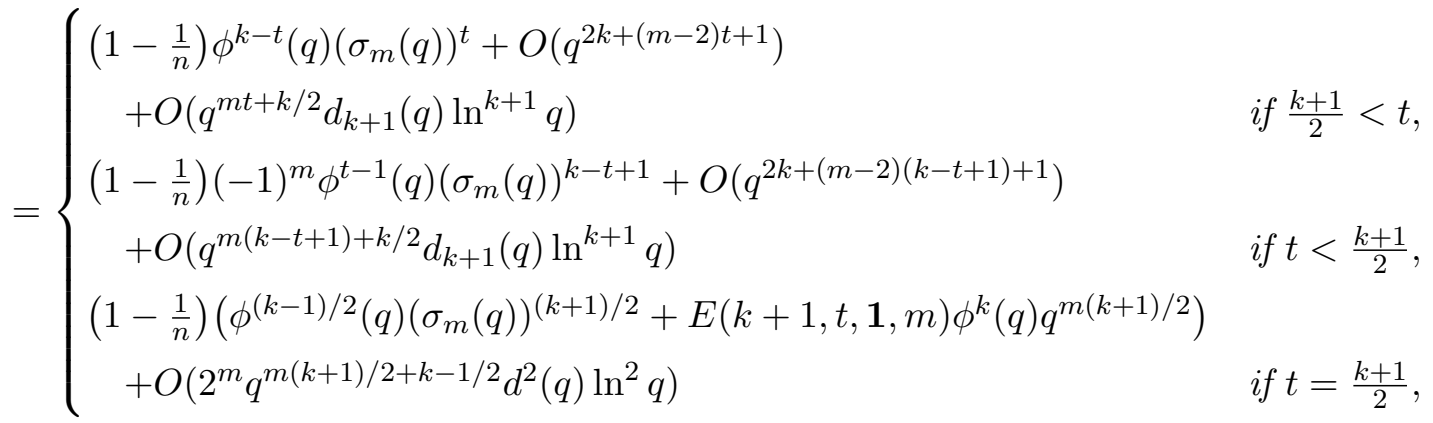

where $\sigma_{m}(q)=\sigma_{m}(1, q)$.

It is obvious to see that the bounds of error terms in the first two cases are sharper than those in Corollary 1.2 .

Taking $t=k \geq 2$ in Corollary 1.6 , we can immediately obtain the following 
Corollary 1.7. For any positive integer $m$, we have

$$
\begin{aligned}
N(a, k+1, k, \mathbf{1}, q, m, n)= & \sum_{\substack{b_{1}=1 \\
b_{1} b_{2} \cdots b_{k+1} \equiv a(\bmod q) \\
n \nmid\left(b_{1}+b_{2}+\cdots+b_{k+1}\right)}}^{q} \sum_{\substack{b_{k+1}=1 \\
\prime} \sum^{q}\left(b_{1} \cdots b_{k}-b_{k+1}\right)^{m}}^{\prime} \\
= & \left(1-\frac{1}{n}\right)\left(\sigma_{m}(q)\right)^{k}+O\left(q^{(m+1 / 2) k} d_{k+1}(q) \ln ^{k+1} q\right) .
\end{aligned}
$$

Taking $m=0$ in Corollary 1.7 , we have

\section{Corollary 1.8.}

$$
\begin{aligned}
N(a, k+1, t, \mathbf{1}, q, 0, n)= & \sum_{\substack{b_{1}=1 \\
b_{1} b_{2} \cdots b_{k+1} \equiv a(\bmod q) \\
n \nmid\left(b_{1}+b_{2}+\cdots+b_{k+1}\right)}}^{q} \sum_{\substack{b_{k+1}=1 \\
q}}^{q} 1 \\
= & \left(1-\frac{1}{n}\right) \phi^{k}(q)+O\left(q^{k / 2} d_{k+1}(q) \ln ^{k+1} q\right) .
\end{aligned}
$$

From the discussions in references [11] and [14], the bound in Corollary 1.8 is close to the best possible. Taking $k=1, n=2$ in Corollary 1.8 , we can immediately obtain the result of $[16]$.

\section{Several lemmas}

To prove Theorems 1.1 and 1.5 , we need the following lemmas.

Lemma 2.1. Let $q$ and $t$ be integers with $q>2$ and $t \geq 0$. Let $y$ and $l$ be integers with $1 \leq y \leq q$ and $1 \leq l \leq n$. Let $0<\lambda \leq 1$ be a real number. For any given integer $n \geq 2$, we have

$$
K(-y, t, l)=\sum_{c=1}^{[\lambda q]} c^{t} \mathrm{e}\left(c \frac{-y n+q l}{q n}\right)= \begin{cases}\frac{(\lambda q)^{t+1}}{t+1}+O\left((\lambda q)^{t}\right) & \text { if } q n \mid(-y n+q l), \\ O\left(\frac{(\lambda q)^{t}}{\left|\sin \frac{\pi(-y n+q l)}{q n}\right|}\right) & \text { if } q n \nmid(-y n+q l) .\end{cases}
$$

Proof. If $q n \nmid(-y n+q l)$, then

$$
\begin{aligned}
& \sum_{c=1}^{[\lambda q]} c^{t} \mathrm{e}\left(c \frac{-y n+q l}{q n}\right)\left(1-\mathrm{e}\left(\frac{-y n+q l}{q n}\right)\right) \\
= & \sum_{c=1}^{[\lambda q]} c^{t} \mathrm{e}\left(c \frac{-y n+q l}{q n}\right)-\sum_{c=1}^{[\lambda q]} c^{t} \mathrm{e}\left(\frac{(c+1)(-y n+q l)}{q n}\right) \\
= & e\left(\frac{-y n+q l}{q n}\right)-[\lambda q]^{t} \mathrm{e}\left(\frac{([\lambda q]+1)(-y n+q l)}{q n}\right)
\end{aligned}
$$




$$
\begin{aligned}
& +\sum_{c=1}^{[\lambda q]-1}(c+1)^{t} \mathrm{e}\left(\frac{(c+1)(-y n+q l)}{q n}\right)-\sum_{c=1}^{[\lambda q]-1} c^{t} \mathrm{e}\left(\frac{(c+1)(-y n+q l)}{q n}\right) \\
& \ll 1+[\lambda q]^{t}+\sum_{c=1}^{[\lambda q]-1}\left((c+1)^{t}-c^{t}\right) \\
& \ll[\lambda q]^{t} .
\end{aligned}
$$

Since

$$
\left|\left(1-\mathrm{e}\left(\frac{-y n+q l}{q n}\right)\right)\right|=\left|2 \sin \frac{\pi(-y n+q l)}{q n}\right|,
$$

we have

$$
K(-y, t, l)=O\left(\frac{(\lambda q)^{t}}{\left|\sin \frac{\pi(-y n+q l)}{q n}\right|}\right) .
$$

If $q n \mid(-y n+q l)$, then we get

$$
K(-y, t, l)=\sum_{c=1}^{[\lambda q]} c^{t}=\frac{(\lambda q)^{t+1}}{t+1}+O\left((\lambda q)^{t}\right) .
$$

This proves Lemma 2.1.

Lemma 2.2. For each prime $p$ and any $\mathbf{y} \in \mathbb{Z}^{k+1}$, there exists a unique integer $r \geq 0$ such that $\mathbf{y}=p^{r} \mathbf{x}$ for some $\mathbf{x} \in \mathbb{Z}^{k+1}-p \mathbb{Z}^{k+1}$. Let $t$ denote the number of components of $\mathbf{x}$ which are divisible by $p$ such that $0 \leq t \leq k$. For each $\alpha \geq 0$, define

$$
\left(\mathbf{y} ; p^{\alpha}\right)_{k}=p^{\sigma_{k}\left(\mathbf{y} ; p^{\alpha}\right)_{k}}
$$

where

$$
\sigma_{k}\left(\mathbf{y} ; p^{\alpha}\right)_{k}= \begin{cases}\alpha & \text { if } r \geq \alpha, \\ r & \text { if } r<\alpha-1, \\ r & \text { if } r=\alpha-1 \text { and } t=0, \\ r-1+\frac{2(t-1)}{k} & \text { if } r=\alpha-1 \text { and } 1 \leq t \leq k .\end{cases}
$$

For any integer $q \geq 1$, we now define

$$
(\mathbf{y} ; q)_{k}=\prod_{p^{\alpha} \| q}\left(\mathbf{y} ; p^{\alpha}\right)_{k}
$$

Then for all integers $k, q \geq 1$ and all $\mathbf{y} \in \mathbb{Z}^{k+1}$, we have the upper bound

$$
\left|S_{k}(\mathbf{y} ; q)\right| \leq q^{k / 2}(\mathbf{y} ; q)_{k}^{k / 2} d_{k+1}(q),
$$

where $d_{k+1}(n)$ is the $k+1$-th divisor function (i.e., the number of solutions of the equation $n_{1} n_{2} \cdots n_{k+1}=n$ in positive integers $\left.n_{1}, n_{2}, \ldots, n_{k+1}\right)$. 
Proof. See 9].

Lemma 2.3. Let $q, k, n$, a be positive integers with $q>2$ and $(n, q)=(a, q)=1$, let $0<$ $\lambda_{1}, \lambda_{2}, \ldots, \lambda_{k+1} \leq 1$ be real numbers. Then for any nonnegative integers $t_{1}, t_{2}, \ldots, t_{k+1}$, we have

$$
\begin{aligned}
\sum_{b_{1}=1}^{\left[\lambda_{1} q\right]} \sum_{b_{2}=1}^{\left[\lambda_{2} q\right]} \cdots \sum_{\substack{b_{1} b_{2} \cdots b_{k+1} \equiv a(\bmod q) \\
b_{2}}}^{\left.\prime \lambda_{k+1} q\right]} b_{1}^{t_{1}} b_{2}^{t_{2}} \cdots b_{k+1}^{t_{k+1}}= & \frac{\lambda_{1}^{t_{1}+1} \lambda_{2}^{t_{2}+1} \cdots \lambda_{k+1}^{t_{k+1}+1} \phi^{k}(q) q^{t_{1}+t_{2}+\cdots+t_{k+1}}}{\left(t_{1}+1\right)\left(t_{2}+1\right) \cdots\left(t_{k+1}+1\right)} \\
& +O\left(q^{t_{1}+t_{2}+\cdots+t_{k+1}+k-1 / 2} d^{2}(q) \ln ^{2} q\right),
\end{aligned}
$$

$\sum_{\substack{b_{1}=1 \\ b_{1} b_{2} \cdots b_{k+1} \equiv a(\bmod q) \\ n \nmid\left(b_{1}+b_{2}+\cdots+b_{k+1}\right)}}^{\left[\lambda_{1} q\right]} \sum_{\substack{\left.b_{k+1}=1 \\ n \lambda_{2} q\right]}}^{\prime} \sum_{1}^{\left.\lambda_{k+1} q\right]} b_{2}^{t_{2}} \cdots b_{k+1}^{t_{k+1}}=\left(1-\frac{1}{n}\right) \frac{\lambda_{1}^{t_{1}+1} \cdots \lambda_{k}^{t_{k}+1} \lambda_{k+1}^{t_{k+1}+1} \phi^{k}(q) q^{t_{1}+t_{2}+\cdots+t_{k+1}}}{\left(t_{1}+1\right)\left(t_{2}+1\right) \cdots\left(t_{k+1}+1\right)}$

$$
+O\left(q^{t_{1}+t_{2}+\cdots+t_{k+1}+k-1 / 2} d^{2}(q) \ln ^{2} q\right),
$$

and

$$
\begin{aligned}
& \sum_{l=1}^{n-1} \sum_{\substack{b_{1}=1 \\
b_{1} b_{2} \cdots b_{k+1} \equiv a(\bmod q)}}^{\left[\lambda_{1} q\right]} \sum_{\substack{\left.b_{k+1}=1 \\
\lambda_{2} q\right]}}^{\prime} \cdots \sum_{\substack{\left.\lambda_{k+1} q\right] \\
=}}^{\prime}\left(\mathrm{e}\left(\frac{b_{1} l}{n}\right) b_{1}^{t_{1}}\right)\left(\mathrm{e}\left(\frac{b_{2} l}{n}\right) b_{2}^{t_{2}}\right) \cdots\left(\mathrm{e}\left(\frac{b_{k+1} l}{n}\right) b_{k+1}^{t_{k+1}}\right) \\
& O\left(q^{t_{1}+t_{2}+\cdots+t_{k+1}+k / 2} d_{k+1}(q) \ln ^{k+1} q\right) .
\end{aligned}
$$

Proof. (i) For the first part of Lemma 2.3, see Lemma 2.6 in [11].

(ii) For the second part, first we have

$$
\begin{aligned}
& \sum_{b_{1}=1}^{\left[\lambda_{1} q\right]} \sum_{b_{2}=1}^{\left.\prime \lambda_{2} q\right]} \cdots \sum_{b_{k+1}=1}^{\prime} b_{1}^{\left.\lambda_{k+1} q\right]} b_{2}^{t_{2}} \cdots b_{k+1}^{t_{k+1}} \\
& b_{1} b_{2} \cdots b_{k+1} \equiv a(\bmod q) \\
& n \nmid\left(b_{1}+b_{2}+\cdots+b_{k+1}\right) \\
& =\sum_{b_{1}=1}^{\left.\prime \lambda_{1} q\right]} \sum_{b_{2}=1}^{\left.\prime \lambda_{2} q\right]} \cdots \sum_{\substack{b_{k+1}=1 \\
b_{1} b_{2} \cdots b_{k+1} \equiv a(\bmod q)}}^{\prime} b_{1}^{t_{1}} b_{2}^{t_{2}} \cdots b_{k+1}^{t_{k+1}}-\sum_{\substack{\lambda_{1}=1 \\
b_{1} b_{2} \cdots b_{k+1} \equiv a(\bmod q) \\
n \mid\left(b_{1}+b_{2}+\cdots+b_{k+1}\right)}}^{\left.\lambda_{b_{1}} q\right]} \sum_{\substack{\left[\lambda_{2} q\right] \\
b_{k+1}=1}}^{\prime} \cdots \sum_{1}^{\left[\lambda_{k+1} q\right]} b_{1}^{t_{1}} b_{2}^{t_{2}} \cdots b_{k+1}^{t_{k+1}} \text {. }
\end{aligned}
$$

Thus we only need to focus on the second sum. By using the trigonometric identity

$$
\sum_{a=1}^{q} \mathrm{e}\left(\frac{a u}{q}\right)= \begin{cases}q & \text { if } q \mid u, \\ 0 & \text { if } q \nmid u,\end{cases}
$$


we obtain

$$
\begin{aligned}
& {\left[\lambda_{1} q\right]\left[\lambda_{2} q\right] \quad\left[\lambda_{k+1} q\right]} \\
& \sum_{b_{1}=1}^{\prime} \sum_{b_{2}=1}^{\prime} \cdots \sum_{b_{k+1}=1}^{\prime} b_{1}^{t_{1}} b_{2}^{t_{2}} \cdots b_{k+1}^{t_{k+1}} \\
& b_{1} b_{2} \cdots b_{k+1} \equiv a(\bmod q) \\
& n \mid\left(b_{1}+b_{2}+\cdots+b_{k+1}\right) \\
& =\frac{1}{n} \sum_{b_{1}=1}^{\left[\lambda_{1} q\right]} \sum_{b_{2}=1}^{\prime} \lambda_{\left.b_{2} q\right]}^{\prime} \cdots \sum_{b_{k+1}=1}^{\left[\lambda_{k+1} q\right]} b_{1}^{t_{1}} b_{2}^{t_{2}} \cdots b_{k+1}^{t_{k+1}} \sum_{l=1}^{n} \mathrm{e}\left(\frac{b_{1}+b_{2}+\cdots+b_{k+1}}{n} l\right) \\
& b_{1} b_{2} \cdots b_{k+1} \equiv a(\bmod q) \\
& =\frac{1}{n q^{k+1}} \sum_{b_{1}=1}^{q} \sum_{b_{2}=1}^{q} \cdots \sum_{b_{k+1}=1}^{\prime} \sum_{c_{1}=1}^{\left[\lambda_{1} q\right]} \cdots \sum_{c_{k}=1}^{\prime} \sum_{c_{k+1}=1}^{\left[\lambda_{k} q\right]} c_{1}^{t_{1}} c_{2}^{t_{2}} \cdots c_{k+1}^{t_{k+1}} \sum_{l=1}^{n} \mathrm{e}\left(\frac{c_{1}+c_{2}+\cdots+c_{k+1}}{n} l\right) \\
& b_{1} b_{2} \cdots b_{k+1} \equiv a(\bmod q) \\
& \times \sum_{y_{1}, \ldots, y_{k+1}=1}^{q} \mathrm{e}\left(\frac{y_{1}\left(b_{1}-c_{1}\right)+\cdots+y_{k}\left(b_{k}-c_{k}\right)+y_{k+1}\left(b_{k+1}-c_{k+1}\right)}{q}\right) \\
& =\frac{1}{n q^{k+1}} \sum_{y_{1}, \ldots, y_{k+1}=1}^{q} \sum_{b_{1}=1}^{q} \sum_{b_{2}=1}^{q} \cdots \sum_{b_{k}=1}^{q} \mathrm{e}\left(\frac{y_{1} b_{1}+\cdots+y_{k} b_{k}+y_{k+1} a \overline{b_{1} \cdots b_{k}}}{q}\right) \\
& \times \sum_{l=1}^{n}\left(\sum_{c_{1}=1}^{\left[\lambda_{1} q\right]} c_{1}^{t_{1}} \mathrm{e}\left(c_{1} \frac{-y_{1} n+q l}{q n}\right)\right) \cdots\left(\sum_{c_{k+1}=1}^{\left[\lambda_{k+1} q\right]} c_{k+1}^{t_{k+1}} \mathrm{e}\left(c_{k+1} \frac{-y_{k+1} n+q l}{q n}\right)\right) \\
& =\frac{1}{n q^{k+1}} \sum_{y_{1}, \ldots, y_{k+1}=1}^{q} \sum_{l=1}^{n} S\left(y_{1}, \ldots, y_{k}, y_{k+1} a ; q\right) K\left(-y_{1}, t_{1}, l\right) \cdots K\left(-y_{k}, t_{k}, l\right) K\left(-y_{k+1}, t_{k+1}, l\right) \\
& =\frac{1}{n q^{k+1}} S(q, \ldots, q, q a ; q) K\left(-q, t_{1}, n\right) \cdots K\left(-q, t_{k}, n\right) K\left(-q, t_{k+1}, n\right) \\
& +\frac{1}{n q^{k+1}} \sum_{l=1}^{n-1} S(q, \ldots, q, q a ; q) K\left(-q, t_{1}, l\right) \cdots K\left(-q, t_{k}, l\right) K\left(-q, t_{k+1}, l\right) \\
& +\sum_{r=1}^{k} \frac{C_{k}^{r}}{q^{k+1}} E_{1}+\sum_{r=1}^{k} \frac{C_{k}^{r-1}}{q^{k+1}} E_{2} \\
& +\frac{1}{n q^{k+1}} \sum_{y_{1}, \ldots, y_{k+1}=1}^{q-1} \sum_{l=1}^{n} S\left(y_{1}, \ldots, y_{k}, y_{k+1} a ; q\right) K\left(-y_{1}, t_{1}, l\right) \cdots K\left(-y_{k}, t_{k}, l\right) \\
& \times K\left(-y_{k+1}, t_{k+1}, l\right) \\
& :=\Sigma_{1}+\Sigma_{2}+\Sigma_{3}+\Sigma_{4}+\Sigma_{5} \text {, }
\end{aligned}
$$

where $C_{k}^{r}=\frac{k !}{r !(k-r) !}(1 \leq r \leq k), K(-y, t, l)$ and $S\left(y_{1}, \ldots, y_{k}, y_{k+1} a ; q\right)=S_{k}(\mathbf{y} ; q)$ are defined on the above, and

$$
E_{1}=\sum_{y_{1}=1}^{q-1} \cdots \sum_{y_{r}=1}^{q-1} \sum_{l=1}^{n} S\left(y_{1}, \ldots, y_{r}, q, \ldots, q, q a ; q\right) K\left(-y_{1}, t_{1}, l\right) \cdots K\left(-y_{r}, t_{r}, l\right)
$$




$$
\begin{aligned}
& \times K\left(-q, t_{r+1}, l\right) \cdots K\left(-q, t_{k}, l\right) K\left(-q, t_{k+1}, l\right), \\
E_{2}= & \sum_{y_{1}=1}^{q-1} \cdots \sum_{y_{r-1}=1}^{q-1} \sum_{y_{k+1}=1}^{q-1} \sum_{l=1}^{n} S\left(y_{1}, \ldots, y_{r-1}, q, \ldots, q, y_{k+1} a ; q\right) \\
& \times K\left(-y_{1}, t_{1}, l\right) \cdots K\left(-y_{r-1}, t_{r-1}, l\right) K\left(-q, t_{r}, l\right) \cdots K\left(-q, t_{k}, l\right) K\left(-y_{k+1}, t_{k+1}, l\right) .
\end{aligned}
$$

For the contribution of $\Sigma_{1}$, from Lemma 2.1, we obtain

$$
\begin{aligned}
\Sigma_{1}= & \frac{1}{n q^{k+1}} S(q, \ldots, q, q a ; q) K\left(-q, t_{1}, n\right) \cdots K\left(-q, t_{k}, n\right) K\left(-q, t_{k+1}, n\right) \\
= & \frac{\phi^{k}(q)}{n q^{k+1}}\left(\frac{\left(\lambda_{1} q\right)^{t_{1}+1}}{t_{1}+1}+O\left(\left(\lambda_{1} q\right)^{t_{1}}\right)\right) \cdots\left(\frac{\left(\lambda_{k} q\right)^{t_{k}+1}}{t_{k}+1}+O\left(\left(\lambda_{k} q\right)^{t_{k}}\right)\right) \\
& \times\left(\frac{\left(\lambda_{k+1} q\right)^{t_{k+1}+1}}{t_{k+1}+1}+O\left(\left(\lambda_{k+1} q\right)^{t_{k+1}}\right)\right. \\
= & \frac{\lambda_{1}^{t_{1}+1} \cdots \lambda_{k}^{t_{k}+1} \lambda_{k+1}^{t_{k+1}+1} \phi^{k}(q) q^{t_{1}+\cdots+t_{k}+t_{k+1}}}{n\left(t_{1}+1\right) \cdots\left(t_{k}+1\right)\left(t_{k+1}+1\right)}+O\left(q^{t_{1}+\cdots+t_{k}+t_{k+1}+k-1}\right) .
\end{aligned}
$$

For the contribution of $\Sigma_{2}$, by applying the Jordan inequality

$$
\frac{2}{\pi} \leq \frac{\sin x}{x} \quad \text { if }|x| \leq \frac{\pi}{2}
$$

we can get the estimate

$$
\begin{aligned}
\Sigma_{2} & =\frac{1}{n q^{k+1}} \sum_{l=1}^{n-1} S(q, \ldots, q, q a ; q) K\left(-q, t_{1}, l\right) \cdots K\left(-q, t_{k}, l\right) K\left(-q, t_{k+1}, l\right) \\
& \ll \frac{\phi^{k}(q)}{n q^{k+1}} \sum_{l=1}^{n-1} \frac{q^{t_{1}}}{\left|\sin \frac{\pi(-q n+q l)}{q n}\right|} \cdots \frac{q^{t_{k}}}{\left|\sin \frac{\pi(-q n+q l)}{q n}\right|} \frac{q^{t_{k+1}}}{\left|\sin \frac{\pi(-q n+q l)}{q n}\right|} \\
& \ll n^{k} q^{t_{1}+\cdots+t_{k}+t_{k+1}-1} .
\end{aligned}
$$

For the contributions of $\Sigma_{3}$ and $\Sigma_{4}$, we need to estimate $E_{1}$ and $E_{2}$. Now we estimate $E_{1}$. Noting that $q n \mid(-y n+q l)$ if and only if $y=q$ and $l=n$, then

$$
\begin{aligned}
E_{1}= & \sum_{y_{1}=1}^{q-1} \ldots \sum_{y_{r}=1}^{q-1} \sum_{l=1}^{n} S\left(y_{1}, \ldots, y_{r}, q, \ldots, q, q a ; q\right) K\left(-y_{1}, t_{1}, l\right) \cdots K\left(-y_{r}, t_{r}, l\right) \\
& \times K\left(-q, t_{r+1}, l\right) \cdots K\left(-q, t_{k}, l\right) K\left(-q, t_{k+1}, l\right) \\
= & \sum_{y_{1}=1}^{q-1} \cdots \sum_{y_{r}=1}^{q-1} S\left(y_{1}, \ldots, y_{r}, q, \ldots, q, q a ; q\right) K\left(-y_{1}, t_{1}, n\right) \cdots K\left(-y_{r}, t_{r}, n\right) \\
& \times K\left(-q, t_{r+1}, n\right) \cdots K\left(-q, t_{k}, n\right) K\left(-q, t_{k+1}, n\right) \\
& +\sum_{y_{1}=1}^{q-1} \cdots \sum_{y_{r}=1}^{q-1} \sum_{l=1}^{n-1} S\left(y_{1}, \ldots, y_{r}, q, \ldots, q, q a ; q\right) K\left(-y_{1}, t_{1}, l\right) \cdots K\left(-y_{r}, t_{r}, l\right)
\end{aligned}
$$




$$
\begin{aligned}
& \times K\left(-q, t_{r+1}, l\right) \cdots K\left(-q, t_{k}, l\right) K\left(-q, t_{k+1}, l\right) \\
& =\sum_{y_{1}=1}^{q-1} \cdots \sum_{y_{r}=1}^{q-1} \sum_{b_{1}=1}^{q} \cdots \sum_{b_{r}=1}^{q} \sum_{b_{r+1}=1}^{q} \cdots \sum_{b_{k}=1}^{q} \mathrm{e}\left(\frac{y_{1} b_{1}+\cdots+y_{r} b_{r}}{q}\right) \\
& \times K\left(-y_{1}, t_{1}, n\right) \cdots K\left(-y_{r}, t_{r}, n\right) K\left(-q, t_{r+1}, n\right) \cdots K\left(-q, t_{k}, n\right) K\left(-q, t_{k+1}, n\right) \\
& +\sum_{y_{1}=1}^{q-1} \cdots \sum_{y_{r}=1}^{q-1} \sum_{b_{1}=1}^{q} \cdots \sum_{b_{r}=1}^{q} \sum_{b_{r+1}=1}^{q} \cdots \sum_{b_{k}=1}^{q} \mathrm{e}\left(\frac{y_{1} b_{1}+\cdots+y_{r} b_{r}}{q}\right) \\
& \times \sum_{l=1}^{n-1} K\left(-y_{1}, t_{1}, l\right) \cdots K\left(-y_{r}, t_{r}, l\right) K\left(-q, t_{r+1}, l\right) \cdots K\left(-q, t_{k}, l\right) K\left(-q, t_{k+1}, l\right) \\
& \ll \phi^{k-r}(q) q^{r / 2} d^{r}(q) \sum_{y_{1}=1}^{q-1} \cdots \sum_{y_{r}=1}^{q-1} \frac{\left(y_{1}, q\right)^{1 / 2} q^{t_{1}}}{\left|\sin \left(\frac{\pi\left(-y_{1} n+q n\right)}{q n}\right)\right|} \cdots \frac{\left(y_{r}, q\right)^{1 / 2} q^{t_{r}}}{\left|\sin \left(\frac{\pi\left(-y_{r} n+q n\right)}{q n}\right)\right|} \\
& \times\left(\frac{\left(\lambda_{r+1} q\right)^{t_{r+1}+1}}{t_{r+1}+1}+O\left(\left(\lambda_{r+1} q\right)^{t_{r+1}}\right)\right) \cdots\left(\frac{\left(\lambda_{k+1} q\right)^{t_{k+1}+1}}{t_{k+1}+1}+O\left(\left(\lambda_{k+1} q\right)^{t_{k+1}}\right)\right) \\
& +\phi^{k-r}(q) q^{r / 2} d^{r}(q) \sum_{y_{1}=1}^{q-1} \cdots \sum_{y_{r}=1}^{q-1} \sum_{l=1}^{n-1} \frac{\left(y_{1}, q\right)^{1 / 2} q^{t_{1}}}{\left|\sin \left(\frac{\pi\left(-y_{1} n+q l\right)}{q n}\right)\right|} \cdots \frac{\left(y_{r}, q\right)^{1 / 2} q^{t_{r}}}{\left|\sin \left(\frac{\pi\left(-y_{r} n+q l\right)}{q n}\right)\right|} \\
& \times \frac{q^{t_{r+1}}}{\left|\sin \left(\frac{\pi(-q n+q l)}{q n}\right)\right|} \cdots \frac{q^{t_{k+1}}}{\left|\sin \left(\frac{\pi(-q n+q l)}{q n}\right)\right|} \\
& \ll q^{t_{1}+\cdots+t_{k}+t_{k+1}+2 k-r / 2+1} d^{r}(q) \sum_{y_{1}=1}^{q-1} \cdots \sum_{y_{r}=1}^{q-1} \frac{\left(y_{1}, q\right)^{1 / 2} \cdots\left(y_{r}, q\right)^{1 / 2}}{y_{1} \cdots y_{r}} \\
& +n^{r} q^{t_{1}+\cdots+t_{k}+t_{k+1}+k+r / 2} d^{r}(q) \sum_{y_{1}=1}^{q-1} \cdots \sum_{y_{r}=1}^{q-1} \sum_{l=1}^{n-1} \frac{\left(y_{1}, q\right)^{1 / 2} \cdots\left(y_{r}, q\right)^{1 / 2}}{\left|-y_{1} n+q l\right| \cdots\left|-y_{r} n+q l\right|} \\
& \ll q^{t_{1}+\cdots+t_{k}+t_{k+1}+2 k-r / 2+1} d^{2 r}(q) \ln ^{r} q \\
& +q^{t_{1}+\cdots+t_{k}+t_{k+1}+k+r / 2} d^{r}(q) \sum_{y_{1}=1}^{q-1} \cdots \sum_{y_{r}=1}^{q-1} \sum_{l=1}^{n-1} \frac{\left(y_{1}, q\right)^{1 / 2} \cdots\left(y_{r}, q\right)^{1 / 2}}{y_{1} \cdots y_{r}} \\
& \ll q^{t_{1}+\cdots+t_{k}+t_{k+1}+2 k-r / 2+1} d^{2 r}(q) \ln ^{r} q .
\end{aligned}
$$

Then we estimate $E_{2}$. From Lemmas 2.1 and 2.2, we have

$$
\begin{aligned}
E_{2}= & \sum_{y_{1}=1}^{q-1} \ldots \sum_{y_{r-1}=1}^{q-1} \sum_{y_{k+1}=1}^{q-1} S\left(y_{1}, \ldots, y_{r-1}, q, \ldots, q, y_{k+1} a ; q\right) \\
& \times K\left(-y_{1}, t_{1}, n\right) \cdots K\left(-y_{r-1}, t_{r-1}, n\right) K\left(-q, t_{r}, n\right) \cdots K\left(-q, t_{k}, n\right) K\left(-y_{k+1}, t_{k+1}, n\right) \\
& +\sum_{y_{1}=1}^{q-1} \ldots \sum_{y_{r-1}=1}^{q-1} \sum_{y_{k+1}=1}^{q-1} \sum_{l=1}^{n-1} S\left(y_{1}, \ldots, y_{r-1}, q, \ldots, q, y_{k+1} a ; q\right) \\
& \times K\left(-y_{1}, t_{1}, l\right) \cdots K\left(-y_{r-1}, t_{r-1}, l\right) K\left(-q, t_{r}, l\right) \cdots K\left(-q, t_{k}, l\right) K\left(-y_{k+1}, t_{k+1}, l\right)
\end{aligned}
$$




$$
\begin{aligned}
& \ll \sum_{y_{1}=1}^{q-1} \cdots \sum_{y_{r-1}=1}^{q-1} \sum_{y_{k+1}=1}^{q-1} q^{k / 2}(\mathbf{y} ; q)_{k}^{k / 2} d_{k+1}(q) \frac{q^{t_{1}}}{\left|\sin \left(\frac{\pi\left(-y_{1} n+q n\right)}{q n}\right)\right|} \cdots \frac{q^{t_{r-1}}}{\left|\sin \left(\frac{\pi\left(-y_{r-1} n+q n\right)}{q n}\right)\right|} \\
& \times \frac{q^{t_{k+1}}}{\left|\sin \left(\frac{\pi\left(-y_{k+1} n+q n\right)}{q n}\right)\right|}\left(\frac{\left(\lambda_{r} q\right)^{t_{r}+1}}{t_{r}+1}+O\left(\left(\lambda_{r} q\right)^{t_{r}}\right)\right) \cdots\left(\frac{\left(\lambda_{k} q\right)^{t_{k}+1}}{t_{k}+1}+O\left(\left(\lambda_{k} q\right)^{t_{k}}\right)\right) \\
& +\sum_{y_{1}=1}^{q-1} \cdots \sum_{y_{r-1}=1}^{q-1} \sum_{y_{k+1}=1}^{q-1} q^{k / 2}(\mathbf{y} ; q)_{k}^{k / 2} d_{k+1}(q) \sum_{l=1}^{n-1} \frac{q^{t_{1}}}{\left|\sin \left(\frac{\pi\left(-y_{1} n+q l\right)}{q n}\right)\right|} \\
& \times \cdots \frac{q^{t_{r-1}}}{\left|\sin \left(\frac{\pi\left(-y_{r-1} n+q l\right)}{q n}\right)\right|} \frac{q^{t_{k+1}}}{\left|\sin \left(\frac{\pi\left(-y_{k+1} n+q l\right)}{q n}\right)\right|} \frac{q^{t_{r}}}{\left|\sin \left(\frac{\pi(-q n+q l)}{q n}\right)\right|} \cdots \frac{q^{t_{k}}}{\left|\sin \left(\frac{\pi(-q n+q l)}{q n}\right)\right|} \\
& \ll q^{t_{1}+\cdots+t_{k}+t_{k+1}+3 k / 2+1} d_{k+1}(q) \sum_{y_{1}=1}^{q-1} \cdots \sum_{y_{r-1}=1}^{q-1} \sum_{y_{k+1}=1}^{q-1} \frac{\left(y_{1}, \ldots, y_{r-1}, y_{k+1}, q\right)^{k / 2}}{y_{1} \cdots y_{r-1} y_{k+1}} \\
& +q^{t_{1}+\cdots+t_{k}+t_{k+1}+k / 2+r} d_{k+1}(q) \\
& \times \sum_{y_{1}=1}^{q-1} \cdots \sum_{y_{r-1}=1}^{q-1} \sum_{y_{k+1}=1}^{q-1} \sum_{l=1}^{n-1} \frac{\left(y_{1}, \ldots, y_{r-1}, y_{k+1}, q\right)^{k / 2}}{\left|-y_{1} n+q l\right| \cdots\left|-y_{r-1} n+q l\right|\left|-y_{k+1} n+q l\right| l^{k-r+1}} \\
& \ll q^{t_{1}+\cdots+t_{k}+t_{k+1}+3 k / 2+1} d_{k+1}(q) \sum_{d \mid q} \sum_{s_{1}=1}^{(q-1) / d} \cdots \sum_{s_{r-1}=1}^{(q-1) / d(q-1) / d} \sum_{s_{r}=1} \frac{d^{(k-2 r) / 2}}{s_{1} \cdots s_{r-1} s_{r}} \\
& +q^{t_{1}+\cdots+t_{k}+t_{k+1}+k / 2+r} d_{k+1}(q) \sum_{y_{1}=1}^{q-1} \cdots \sum_{y_{r-1}=1}^{q-1} \sum_{y_{k+1}=1}^{q-1} \sum_{l=1}^{n-1} \frac{\left(y_{1}, \ldots, y_{r-1}, y_{k+1}, q\right)^{k / 2}}{y_{1} \cdots y_{r-1} y_{k+1} n^{r} l^{k-r+1}} \\
& \ll q^{t_{1}+\cdots+t_{k}+t_{k+1}+2 k-r+1} d_{k+1}(q) d(q) \ln ^{r} q .
\end{aligned}
$$

Finally, for the contribution of $\Sigma_{5}$, we have

$$
\begin{aligned}
\Sigma_{5}= & \frac{1}{n q^{k+1}} \sum_{y_{1}, \ldots, y_{k+1}=1}^{q-1} \sum_{l=1}^{n} S\left(y_{1}, \ldots, y_{k}, y_{k+1} a ; q\right) \\
& \times K\left(-y_{1}, t_{1}, l\right) \cdots K\left(-y_{k}, t_{k}, l\right) K\left(-y_{k+1}, t_{k+1}, l\right) \\
\ll & \frac{1}{n q^{k+1}} \sum_{y_{1}=1}^{q-1} \cdots \sum_{y_{k}=1}^{q-1} \sum_{y_{k+1}=1}^{q-1} \sum_{l=1}^{n} q^{k / 2}(\mathbf{y} ; q)_{k}^{k / 2} d_{k+1}(q) \\
& \times \frac{\left(\lambda_{1} q\right)^{t_{1}}}{\left|\sin \left(\frac{\pi\left(-y_{1} n+q l\right)}{q n}\right)\right|} \cdots \frac{\left(\lambda_{k} q\right)^{t_{k}}}{\left|\sin \left(\frac{\pi\left(-y_{k} n+q l\right)}{q n}\right)\right|\left|\sin \left(\frac{\pi\left(-y_{k+1} n+q l\right)}{q n}\right)\right|} \\
\ll & q^{t_{1}+\cdots+t_{k}+t_{k+1}+k / 2} d_{k+1}(q) \sum_{y_{1}=1}^{q-1} \cdots \sum_{y_{k}=1}^{q-1} \sum_{y_{k+1}=1}^{q-1} \\
& \times \sum_{l=1}^{n} \frac{\left(y_{1}, \ldots, y_{k}, y_{k+1}, q\right)^{k / 2} n^{k+1}}{\left|-y_{1} n+q l\right| \cdots\left|-y_{k} n+q l\right|\left|-y_{k+1} n+q l\right|}
\end{aligned}
$$




$$
\begin{aligned}
& \ll q^{t_{1}+\cdots+t_{k}+t_{k+1}+k / 2} d_{k+1}(q) \sum_{y_{1}=1}^{q-1} \cdots \sum_{y_{k}=1}^{q-1} \sum_{y_{k+1}=1}^{q-1} \sum_{l=1}^{n} \frac{\left(y_{1}, \ldots, y_{k}, y_{k+1}, q\right)^{k / 2}}{y_{1} \cdots y_{k} y_{k+1}} \\
& \ll q^{t_{1}+\cdots+t_{k}+t_{k+1}+k / 2} d_{k+1}(q) \sum_{d \mid q} \sum_{s_{1}=1}^{(q-1) / d} \cdots \sum_{s_{k}=1}^{(q-1) / d} \sum_{s_{k+1}=1}^{(q-1) / d} \frac{1}{d^{(k+2) / 2} s_{1} \cdots s_{k} s_{k+1}} \\
& \ll q^{t_{1}+\cdots+t_{k}+t_{k+1}+k / 2} d_{k+1}(q) \ln ^{k+1} q .
\end{aligned}
$$

Collecting the contributions of all terms, we immediately deduce

$$
\begin{aligned}
& \sum_{b_{1}=1}^{\left[\lambda_{1} q\right]} \sum_{b_{2}=1}^{\left[\lambda_{2} q\right]} \cdots \sum_{\substack{b_{k+1}=1 \\
b_{1} b_{2} \cdots b_{k+1} \\
n \mid\left(b_{1}+b_{2}+\cdots+b_{k+1}\right)}}^{\left[\lambda_{k+1} q\right]} b_{1}^{t_{1}} b_{2}^{t_{2}} \cdots b_{k+1}^{t_{k+1}} \\
= & \frac{\lambda_{1}^{t_{1}+1} \cdots \lambda_{k}^{t_{k}+1} \lambda_{k+1}^{t_{k+1}+1} \phi^{k}(q) q^{t_{1}+\cdots+t_{k}+t_{k+1}}}{n\left(t_{1}+1\right) \cdots\left(t_{k}+1\right)\left(t_{k+1}+1\right)}+O\left(q^{t_{1}+\cdots+t_{k}+t_{k+1}+k-1 / 2} d^{2}(q) \ln ^{2} q\right),
\end{aligned}
$$

which implies the second part of Lemma 2.3 .

(iii) For the third part of Lemma 2.3. similarly, we have

$$
\begin{aligned}
& \sum_{l=1}^{n-1} \sum_{\substack{b_{1}=1 \\
b_{1} b_{2} \cdots b_{k+1} \equiv a(\bmod q)}}^{\left[\lambda_{1} q\right]} \sum_{\substack{\left.b_{2}=1 \\
\lambda_{2} q\right]}}^{\prime} \sum_{\substack{k+1 \\
k+1}}^{\left.\prime \lambda_{k+1} q\right]}\left(\mathrm{e}\left(\frac{b_{1} l}{n}\right) b_{1}^{t_{1}}\right)\left(\mathrm{e}\left(\frac{b_{2} l}{n}\right) b_{2}^{t_{2}}\right) \cdots\left(\mathrm{e}\left(\frac{b_{k+1} l}{n}\right) b_{k+1}^{t_{k+1}}\right) \\
& =\frac{1}{q^{k+1}} \sum_{b_{1}=1}^{q} \cdots \sum_{b_{k}=1}^{\prime} \sum_{b_{k+1}=1}^{\prime} \sum_{c_{1}=1}^{\lambda_{1} q} \cdots \sum_{c_{k}=1}^{\lambda_{k} q} \sum_{c_{k+1}=1}^{\lambda_{k+1} q}\left(\mathrm{e}\left(\frac{c_{1} l}{n} c_{1}^{t_{1}}\right)\right) \cdots\left(\mathrm{e}\left(\frac{c_{k+1} l}{n} c_{k+1}^{t_{k+1}}\right)\right) \\
& b_{1} \cdots b_{k} b_{k+1} \equiv a(\bmod q) \\
& \times \sum_{y_{1}, \ldots, y_{k}, y_{k+1}=1}^{q} \mathrm{e}\left(\frac{y_{1}\left(b_{1}-c_{1}\right)+\cdots+y_{k}\left(b_{k}-c_{k}\right)+y_{k+1}\left(b_{k+1}-c_{k+1}\right)}{q}\right) \\
& =\frac{1}{q^{k+1}} \sum_{y_{1}, \ldots, y_{k}, y_{k+1}=1}^{q} \sum_{b_{1}=1}^{q} \cdots \sum_{b_{k}=1}^{q} \mathrm{e}\left(\frac{y_{1} b_{1}+\cdots+y_{k} b_{k}+y_{k+1} a \overline{b_{1} \cdots b_{k}}}{q}\right) \\
& \times \sum_{l=1}^{n-1}\left(\sum_{c_{1}=1}^{\lambda_{1} q} c_{1}^{t_{1}} \mathrm{e}\left(c_{1} \frac{-y_{1} n+q l}{q n}\right)\right) \cdots\left(\sum_{c_{k}=1}^{\lambda_{k} q} c_{k}^{t_{k}} \mathrm{e}\left(c_{k} \frac{-y_{k} n+q l}{q n}\right)\right) \\
& \times\left(\sum_{c_{k+1}=1}^{\lambda_{k+1} q} c_{k+1}^{t_{k+1}} \mathrm{e}\left(c_{k+1} \frac{-y_{k+1} n+q l}{q n}\right)\right) \\
& =\frac{1}{q^{k+1}} \sum_{y_{1}, \ldots, y_{k}, y_{k+1}=1}^{q} \sum_{l=1}^{n-1} S\left(y_{1}, \ldots, y_{k}, y_{k+1} a ; q\right) \\
& \times K\left(-y_{1}, t_{1}, l\right) \cdots K\left(-y_{k}, t_{k}, l\right) K\left(-y_{k+1}, t_{k+1}, l\right) .
\end{aligned}
$$


Then using the same method as above, we have

$$
\begin{aligned}
& \sum_{\substack{l=1 \\
b_{1}=1 \\
b_{1} b_{2} \cdots b_{k+1} \equiv a(\bmod q)}}^{n-1} \sum_{b_{2}=1}^{\left[\lambda_{1} q\right]} \cdots \sum_{b_{k+1}=1}^{\left[\lambda_{2} q\right]}\left(\mathrm{e}\left(\frac{b_{1} l}{n}\right) b_{1}^{t_{1}}\right)\left(\mathrm{e}\left(\frac{b_{2} l}{n}\right) b_{2}^{t_{2}}\right) \cdots\left(\mathrm{e}\left(\frac{b_{k+1} l}{n}\right) b_{k+1}^{t_{k+1}}\right) \\
= & O\left(q^{t_{1}+t_{2}+\cdots+t_{k+1}+k / 2} d_{k+1}(q) \ln ^{k+1} q\right) .
\end{aligned}
$$

This proves Lemma 2.3 .

Lemma 2.4. Let $q, k, t, m$ be positive integers with $q>2$, and $a$ an integer with $(a, q)=1$. Then

$$
\sum_{\substack{b_{1}=1 \\ b_{1} \cdots b_{k} b_{k+1} \equiv a(\bmod q)}}^{\left[\lambda_{1} q\right]} \sum_{\substack{b_{k}=1 \\ b_{k+1}=1}}^{\left[\lambda_{k} q\right]} \sum_{j=1}^{q} C_{m}^{j}\left(b_{1} \cdots b_{t}\right)^{m-j}\left(b_{t+1} \cdots b_{k+1}\right)^{j}=O\left(q^{2 k+(m-2) t+1}\right)
$$

if $(k+1) / 2<t<k+1$, and

$$
\sum_{\substack{b_{1}=1 \\ b_{1} \cdots b_{k} b_{k+1} \equiv a(\bmod q)}}^{q} \cdots \sum_{\substack{b_{k}=1 \\ b_{k+1}=1}}^{\left[\lambda_{k} q\right]} \sum_{j=1}^{\left[\lambda_{k+1} q\right]} C_{m}^{j}\left(b_{1} \cdots b_{t}\right)^{j}\left(b_{t+1} \cdots b_{k+1}\right)^{m-j}=O\left(q^{2 k+(m-2)(k-t+1)+1}\right)
$$

if $t<(k+1) / 2$.

Proof. Note that

$$
\left(1+q^{k-2 t+1}\right)^{m}-1=O\left(m q^{k-2 t+1}\right)
$$

holds for $t>(k+1) / 2$, we have

$$
\begin{aligned}
& \sum_{\substack{b_{1}=1 \\
b_{1} \cdots b_{k} b_{k+1} \equiv a(\bmod q)}}^{\left[\lambda_{1} q\right]} \ldots \sum_{b_{k}=1}^{\left[\lambda_{k} q\right]} \sum_{b_{k+1}=1}^{q} \sum_{j=1}^{m} C_{m}^{j}\left(b_{1} \cdots b_{t}\right)^{m-j}\left(b_{t+1} \cdots b_{k+1}\right)^{j} \\
= & O\left(\sum_{b_{1}=1}^{\left[\lambda_{1} q\right]} \sum_{b_{1} \cdots b_{k} b_{k+1} \equiv a(\bmod q)}^{\prime} \ldots \sum_{\left.b_{k} q\right]}^{\prime} \sum_{b_{k+1}=1}^{q} \sum_{j=1}^{m} C_{m}^{j} q^{t(m-j)+(k-t+1) j}\right) \\
= & O\left(q^{m t} \sum_{j=1}^{m} C_{m}^{j} q^{(k-2 t+1) j} \sum_{b_{1}=1}^{\prime} \cdots \sum_{b_{k}=1}^{\left[\lambda_{k}\right.} 1\right) \\
= & O\left(q^{m t}\left(\left(1+q^{k-2 t+1}\right)^{m}-1\right) \prod_{u=1}^{k} \sigma_{0}\left(\lambda_{u}, q\right)\right) \\
= & O\left(q^{2 k+(m-2) t+1}\right) .
\end{aligned}
$$


If $t<(k+1) / 2$, similarly we have

$$
\sum_{\substack{b_{1}=1 \\ b_{1} \cdots b_{k} b_{k+1} \equiv a(\bmod q)}}^{q} \cdots \sum_{\substack{\left.b_{k}=1 \\ \lambda_{k} q\right]}}^{\left[\lambda_{k+1}\right]} \sum_{j=1}^{\left[\lambda_{k+1} q\right]} C_{m}^{j}\left(b_{1} \cdots b_{t}\right)^{j}\left(b_{t+1} \cdots b_{k+1}\right)^{m-j}=O\left(q^{2 k+(m-2)(k-t+1)+1}\right) .
$$

This completes the proof of Lemma 2.4

\section{Proofs of theorems}

Proof of Theorem 1.1. First for $m>0$, we have

$$
\begin{aligned}
& N(a, k+1, t, \mathbf{w}, q, m, n) \\
& =\sum_{b_{1}=1}^{\left[\lambda_{1} q\right]} \sum_{b_{2}=1}^{\prime} \cdots \sum_{b_{k+1}=1}^{\left[\lambda_{2} q\right]}\left(b_{1} \cdots b_{t}-b_{t+1} \cdots b_{k+1}\right)^{m} \\
& b_{1} b_{2} \cdots b_{k+1} \equiv a(\bmod q) \\
& n \nmid\left(b_{1}+b_{2}+\cdots+b_{k+1}\right) \\
& =\sum_{j=0}^{m} C_{m}^{j}(-1)^{j} \sum_{\substack{b_{1}=1 \\
b_{1} b_{2} \cdots b_{k+1} \equiv a(\bmod q)}}^{\left[\lambda_{1} q\right]} \sum_{\substack{\left.b_{2}=1 \\
\lambda_{2} q\right]}}^{\prime} \sum_{\substack{\left.\lambda_{k+1} q\right]\\
}}^{\prime}\left(b_{1} \cdots b_{t}\right)^{m-j}\left(b_{t+1} \cdots b_{k+1}\right)^{j} \\
& =\sum_{j=0}^{m} C_{m}^{j}(-1)^{j}\left(\left(1-\frac{1}{n}\right) \frac{\left(\lambda_{1} \cdots \lambda_{t}\right)^{m-j+1}\left(\lambda_{t+1} \cdots \lambda_{k+1}\right)^{j+1}}{(m-j+1)^{t}(j+1)^{k-t+1}} \phi^{k}(q) q^{m t+(k-2 t+1) j}\right. \\
& \left.+O\left(q^{m t+(k-2 t+1) j+k-1 / 2} d^{2}(q) \ln ^{2} q\right)\right),
\end{aligned}
$$

where we have used Lemma 2.3 .

We can separate it into three cases $t>(k+1) / 2, t<(k+1) / 2$, and $t=(k+1) / 2$. If $t>(k+1) / 2$, noting the fact that the main term only comes from the item $j=0$ and the other items $0<j \leq m$ are all error terms, we have

$$
\begin{aligned}
& N(a, k+1, t, \mathbf{w}, q, m, n) \\
= & \left(1-\frac{1}{n}\right) \frac{\left(\lambda_{1} \cdots \lambda_{t}\right)^{m+1}\left(\lambda_{t+1} \cdots \lambda_{k+1}\right)}{(m+1)^{t}} \phi^{k}(q) q^{m t}+O\left(q^{m t+k-1 / 2} d^{2}(q) \ln ^{2} q\right) .
\end{aligned}
$$

If $t<(k+1) / 2$, similarly we have

$$
\begin{aligned}
N(a, k+1, t, \mathbf{w}, q, m, n)= & \left(1-\frac{1}{n}\right) \frac{(-1)^{m}\left(\lambda_{1} \cdots \lambda_{t}\right)\left(\lambda_{t+1} \cdots \lambda_{k+1}\right)^{m+1}}{(m+1)^{k-t+1}} \phi^{k}(q) q^{m(k-t+1)} \\
& +O\left(q^{m(k-t+1)+k-1 / 2} d^{2}(q) \ln ^{2} q\right) .
\end{aligned}
$$


While if $t=(k+1) / 2$, for the reason that all items $0 \leq j \leq m$ are the main terms, we have

$$
\begin{aligned}
& N(a, k+1, t, \mathbf{w}, q, m, n) \\
= & \sum_{j=0}^{m} C_{m}^{j}(-1)^{j}\left(1-\frac{1}{n}\right) \frac{\left(\lambda_{1} \cdots \lambda_{t}\right)^{m-j+1}\left(\lambda_{t+1} \cdots \lambda_{k+1}\right)^{j+1}}{((m-j+1)(j+1))^{(k+1) / 2}} \phi^{k}(q) q^{m t} \\
& +O\left(2^{m} q^{m t+k-1 / 2} d^{2}(q) \ln ^{2} q\right) .
\end{aligned}
$$

Then for $m=0$, we have

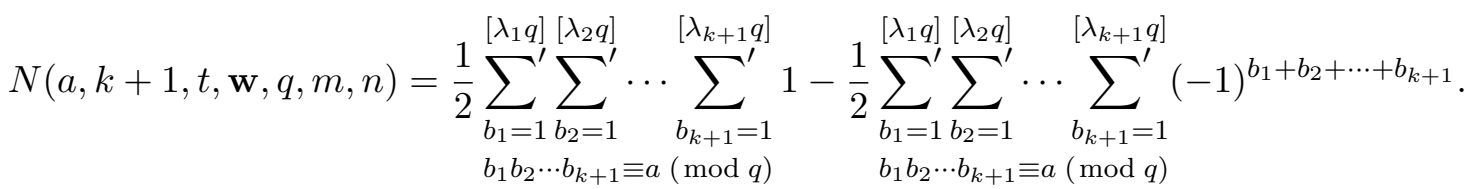

Thus Theorem 1.1 holds again by appealing to Lemma 2.3 . This completes the proof of Theorem 1.1

Proof of Theorem 1.5. Noting that $(k+1) / 2<t<k+1$ and $\mathbf{w}=\left(\lambda_{1}, \lambda_{2}, \ldots, \lambda_{k+1}\right)$ with at least one $\lambda_{j}=1(j>t)$, we can assume $\lambda_{k+1}=1$ (without loss of generality). Then we have

$$
\begin{aligned}
& N_{k+1}(a, k+1, t, \mathbf{w}, q, m, n) \\
& =\sum_{\substack{b_{1}=1 \\
b_{1} b_{2} \cdots b_{k+1} \equiv a(\bmod q) \\
n \nmid\left(b_{1}+b_{2}+\cdots+b_{k+1}\right)}}^{\left[\lambda_{1} q\right]} \cdots \sum_{\substack{b_{k}=1 \\
b_{k+1}=1}}^{\left[\lambda_{k} q\right]} \sum^{q}\left(b_{1} \cdots b_{t}-b_{t+1} \cdots b_{k+1}\right)^{m} \\
& =\sum_{b_{1}=1}^{\left[\lambda_{1} q\right]} \cdots \sum_{b_{k}=1}^{\left[\lambda_{k} q\right]} \sum_{b_{k+1}=1}^{q}\left(b_{1} \cdots b_{t}-b_{t+1} \cdots b_{k+1}\right)^{m} \\
& b_{1} b_{2} \cdots b_{k+1} \equiv a(\bmod q) \\
& -\sum_{b_{1}=1}^{\left[\lambda_{1} q\right]} \cdots \sum_{b_{k}=1}^{\left[\lambda_{k} q\right]} \sum_{b_{k+1}=1}^{q}\left(b_{1} \cdots b_{t}-b_{t+1} \cdots b_{k+1}\right)^{m} \\
& b_{1} b_{2} \cdots b_{k+1} \equiv a(\bmod q) \\
& n \mid\left(b_{1}+b_{2}+\cdots+b_{k+1}\right) \\
& =\sum_{b_{1}=1}^{\left[\lambda_{1} q\right]} \cdots \sum_{b_{k}=1}^{\left[\lambda_{k} q\right]} \sum_{b_{k+1}=1}^{q}\left(b_{1} \cdots b_{t}-b_{t+1} \cdots b_{k+1}\right)^{m} \\
& b_{1} b_{2} \cdots b_{k+1} \equiv a(\bmod q) \\
& -\frac{1}{n} \sum_{b_{1}=1}^{\left[\lambda_{1} q\right]} \cdots \sum_{b_{k}=1}^{\left[\lambda_{k} q\right]} \sum_{b_{k+1}=1}^{q}\left(b_{1} \cdots b_{t}-b_{t+1} \cdots b_{k+1}\right)^{m} \\
& b_{1} b_{2} \cdots b_{k+1} \equiv a(\bmod q)
\end{aligned}
$$




$$
\begin{aligned}
& \left.-\frac{1}{n} \sum_{\substack{b_{1}=1 \\
b_{1} b_{2} \cdots b_{k+1} \equiv a(\bmod q)}}^{\left[\lambda_{1} q\right]} \sum_{\substack{b_{k}=1 \\
b_{k+1}=1}}^{\left[\lambda_{k} q\right]} \sum^{q} b_{1} \cdots b_{t}-b_{t+1} \cdots b_{k+1}\right)^{m} \sum_{l=1}^{n-1} \mathrm{e}\left(\frac{b_{1}+\cdots+b_{k+1}}{n} l\right) \\
& =\left(1-\frac{1}{n}\right) \sum_{b_{1}=1}^{\left[\lambda_{1} q\right]} \cdots \sum_{b_{k}=1}^{\left.\prime \lambda_{k} q\right]} \sum_{b_{k+1}=1}^{q}\left(b_{1} \cdots b_{t}\right)^{m} \\
& b_{1} \cdots b_{k} b_{k+1} \equiv a(\bmod q) \\
& +\left(1-\frac{1}{n}\right) \sum_{j=1}^{m} C_{m}^{j}(-1)^{j} \sum_{b_{1}=1}^{\left[\lambda_{1} q\right]} \cdots \sum_{b_{k}=1}^{\left[\lambda_{k} q\right]} \sum_{b_{k+1}=1}^{q}\left(b_{1} \cdots b_{t}\right)^{m-j}\left(b_{t+1} \cdots b_{k+1}\right)^{j} \\
& -\frac{1}{n} \sum_{j=0}^{m} C_{m}^{j}(-1)^{j} \sum_{l=1}^{n-1} \sum_{b_{1}=1}^{\left[\lambda_{1} q\right]} \cdots \sum_{b_{k}=1}^{\left.\prime \lambda_{k} q\right]} \sum_{b_{k+1}=1}^{q}\left(\mathrm{e}\left(\frac{b_{1} l}{n}\right) b_{1}^{m-j}\right) \cdots\left(\mathrm{e}\left(\frac{b_{t} l}{n}\right) b_{t}^{m-j}\right) \\
& b_{1} \cdots b_{k} b_{k+1} \equiv a(\bmod q) \\
& \times\left(\mathrm{e}\left(\frac{b_{t+1} l}{n}\right) b_{t+1}^{j}\right) \cdots\left(\mathrm{e}\left(\frac{b_{k+1} l}{n}\right) b_{k+1}^{j}\right) \\
& =\left(1-\frac{1}{n}\right) \sum_{b_{1}=1}^{\left[\lambda_{1} q\right]} \cdots \sum_{b_{k}=1}^{\left[\lambda_{k} q\right]}\left(b_{1} \cdots b_{t}\right)^{m}+O\left(q^{m t+k / 2} d_{k+1}(q) \ln ^{k+1} q\right) \\
& +O\left(\sum_{\substack{b_{1}=1 \\
b_{1} \cdots b_{k} b_{k+1} \equiv a(\bmod q)}}^{\left[\lambda_{b_{k}=1}\right.} \sum_{b_{k+1}=1}^{\left[\lambda_{k} q\right]} \sum_{j=1}^{q} C_{m}^{j}\left(b_{1} \cdots b_{t}\right)^{m-j}\left(b_{t+1} \cdots b_{k+1}\right)^{j}\right) \\
& =\left(1-\frac{1}{n}\right) \prod_{u=1}^{t} \sigma_{m}\left(\lambda_{u}, q\right) \prod_{\substack{v=t+1 \\
v \neq j}}^{k+1} \sigma_{0}\left(\lambda_{v}, q\right)+O\left(q^{2 k+(m-2) t+1}\right)+O\left(q^{m t+k / 2} d_{k+1}(q) \ln ^{k+1} q\right),
\end{aligned}
$$

where we have used Lemmas 2.3 and 2.4

If $t<(k+1) / 2$ and $\mathbf{w}=\left(\lambda_{1}, \lambda_{2}, \ldots, \lambda_{k+1}\right)$ with at least one $\lambda_{j}=1(j<t)$, similarly we have

$$
\begin{aligned}
N_{1}(a, k+1, t, \mathbf{w}, q, m, n)= & \left(1-\frac{1}{n}\right)(-1)^{m} \prod_{\substack{u=1 \\
u \neq j}}^{t} \sigma_{0}\left(\lambda_{u}, q\right) \prod_{v=t+1}^{k+1} \sigma_{m}\left(\lambda_{v}, q\right) \\
& +O\left(q^{2 k+(m-2)(k-t+1)+1}\right)+O\left(q^{m(k-t+1)+k / 2} d_{k+1}(q) \ln ^{k+1} q\right) .
\end{aligned}
$$

If $t=(k+1) / 2$ and $\mathbf{w}=\left(\lambda_{1}, \lambda_{2}, \ldots, \lambda_{k+1}\right)$ with at least one $\lambda_{j}=1(j>t)$, we have

$$
\begin{aligned}
& N_{k+1}(a, k+1, t, \mathbf{w}, q, m, n) \\
= & \left(1-\frac{1}{n}\right) \sum_{\substack{b_{1}=1 \\
b_{1} \cdots b_{k} b_{k+1} \equiv a(\bmod q)}}^{\left.\prime \lambda_{1} q\right]} \sum_{\substack{\left.b_{k}=1 \\
\lambda_{k} q\right]}}^{\prime} \sum_{\substack{k+1 \\
\text { mod }}}^{\prime}\left(b_{1} \cdots b_{t}\right)^{m}
\end{aligned}
$$




$$
\begin{aligned}
& +\left(1-\frac{1}{n}\right) \sum_{j=1}^{m} C_{m}^{j}(-1)^{j} \sum_{b_{1}=1}^{\left[\lambda_{1} q\right]} \cdots \sum_{b_{k}=1}^{\left[\lambda_{k} q\right]} \sum_{b_{k+1}=1}^{q}\left(b_{1} \cdots b_{t}\right)^{m-j}\left(b_{t+1} \cdots b_{k+1}\right)^{j} \\
& b_{1} \cdots b_{k} b_{k+1} \equiv a(\bmod q) \\
& -\frac{1}{n} \sum_{j=0}^{m} C_{m}^{j}(-1)^{j} \sum_{l=1}^{n-1} \sum_{b_{1}=1}^{\left[\lambda_{1} q\right]} \cdots \sum_{b_{k}=1}^{\left[\lambda_{k} q\right]} \sum_{b_{k+1}=1}^{q}\left(\mathrm{e}\left(\frac{b_{1} l}{n}\right) b_{1}^{m-j}\right) \cdots\left(\mathrm{e}\left(\frac{b_{t} l}{n}\right) b_{t}^{m-j}\right) \\
& b_{1} \cdots b_{k} b_{k+1} \equiv a(\bmod q) \\
& \times\left(\mathrm{e}\left(\frac{b_{t+1} l}{n}\right) b_{t+1}^{j}\right) \cdots\left(\mathrm{e}\left(\frac{b_{k+1} l}{n}\right) b_{k+1}^{j}\right) \\
& =\left(1-\frac{1}{n}\right) \sum_{b_{1}=1}^{\left[\lambda_{1} q\right]} \cdots \sum_{b_{k}=1}^{\left[\lambda_{k} q\right]}\left(b_{1} \cdots b_{t}\right)^{m}+O\left(q^{m t+k / 2} d_{k+1}(q) \ln ^{k+1} q\right) \\
& +\left(1-\frac{1}{n}\right) \sum_{j=1}^{m} C_{m}^{j}(-1)^{j}\left(\frac{\left(\lambda_{1} \cdots \lambda_{t}\right)^{m-j+1}\left(\lambda_{t+1} \cdots \lambda_{k}\right)^{j+1}}{(m-j+1)^{t}(j+1)^{k-t+1}} \phi^{k}(q) q^{m t+(k-2 t+1) j}\right. \\
& \left.+O\left(q^{m t+(k-2 t+1) j+k-1 / 2} d^{2}(q) \ln ^{2} q\right)\right) \\
& =\left(1-\frac{1}{n}\right) \prod_{u=1}^{(k+1) / 2} \sigma_{m}\left(\lambda_{u}, q\right) \prod_{\substack{v=(k+3) / 2 \\
v \neq j}}^{k+1} \sigma_{0}\left(\lambda_{v}, q\right)+O\left(2^{m} q^{m(k+1) / 2+k-1 / 2} d^{2}(q) \ln ^{2} q\right) \\
& +\left(1-\frac{1}{n}\right) \sum_{j=0}^{m} C_{m}^{j}(-1)^{j} \frac{\left(\lambda_{1} \cdots \lambda_{t}\right)^{m-j+1}\left(\lambda_{t+1} \cdots \lambda_{k}\right)^{j+1}}{((m-j+1)(j+1))^{(k+1) / 2}} \phi^{k}(q) q^{m(k+1) / 2},
\end{aligned}
$$

where we have used Lemmas 2.3 and 2.4 . This completes the proof of Theorem 1.5 .

\section{Acknowledgments}

The authors are grateful to the anonymous referee for very helpful and detailed comments. The authors are also indebted to Professor Igor E. Shparlinski for his kind suggestions and nice reference of 8 .

\section{References}

[1] E. Alkan, F. Stan and A. Zaharescu, Lehmer k-tuples, Proc. Amer. Math. Soc. 134 (2006), no. 10, 2807-2815.

[2] S. D. Cohen and T. Trudgian, Lehmer numbers and primitive roots modulo a prime, J. Number Theory 203 (2019), 68-79.

[3] R. K. Guy, Unsolved Problems in Number Theory, Second edition, Springer-Verlag, New York, 1994. 
[4] M. R. Khan, An optimization with a modular constraint: 10736, Amer. Math. Monthly 108 (2001), no. 4, 374-375.

[5] M. R. Khan and I. E. Shparlinski, On the maximal difference between an element and its inverse modulo n, Period. Math. Hungar. 47 (2003), no. 1-2, 111-117.

[6] Y. M. Lu and Y. Yi, On the generalization of the D. H. Lehmer problem, Acta Math. Sin. (Engl. Ser.) 25 (2009), no. 8, 1269-1274.

[7] _ On the generalization of the D. H. Lehmer problem II, Acta Arith. 142 (2010), no. 2, 179-186.

[8] I. E. Shparlinski, On the distribution of points on multidimensional modular hyperbolas, Proc. Japan Acad. Ser. A Math. Sci. 83 (2007), no. 2, 5-9.

[9] R. A. Smith, On n-dimensional Kloosterman sums, J. Number Theory 11 (1979), no. 3, S. Chowla Anniversary Issue, 324-343.

[10] Z. Xu, Distribution of the difference of an integer and its $m$-th power $\bmod n$ over incomplete intervals, J. Number Theory 133 (2013), no. 12, 4200-4223.

[11] Z. F. Xu and T. P. Zhang, High-dimensional D. H. Lehmer problem over short intervals, Acta Math. Sin. (Engl. Ser.) 30 (2014), no. 2, 213-228.

[12] Z. Xu and W. Zhang, On a problem of D. H. Lehmer over short intervals, J. Math. Anal. Appl. 320 (2006), no. 2, 756-770.

[13] G. T. Zhang and H. N. Liu, On the difference between the non-negative least residue and Fermat quotient of an integer, Journal of Shaanxi Normal University (Natural Science Edition) 45 (2017), no. 04, 11-13.

[14] T. Zhang, High-dimensional D. H. Lehmer problem over quarter intervals, Abstr. Appl. Anal. 2014 (2014), Art. ID 943794, 10 pp.

[15] T. Zhang and W. Zhang, A generalization on the difference between an integer and its inverse modulo q II, Proc. Japan Acad. Ser. A Math. Sci. 81 (2005), no. 1, 7-11.

[16] W. P. Zhang, A problem of D. H. Lehmer and its generalization II, Compositio Math. 91 (1994), no. 1, 47-56.

[17] - On the difference between a D. H. Lehmer number and its inverse modulo q, Acta Arith. 68 (1994), no. 3, 255-263. 
Di Han and Zhefeng Xu

Research Center for Number Theory and Its Applications, Northwest University, Xi'an 710127, Shaanxi, China

E-mail addresses: dhan@nwu.edu.cn, zfxu@nwu.edu.cn

Yuan Yi

School of Mathematics and Statistics, Xi'an Jiaotong University, Xi'an 710049, Shaanxi, China

E-mail address: yuanyi@mail.xjtu.edu.cn

Tianping Zhang

School of Mathematics and Statistics, Shaanxi Normal University, Xi'an 710119, Shaanxi, China

E-mail address: tpzhang@snnu.edu.cn 